Información Tecnológica

Vol. 25(3), 167-176 (2014)

doi: $10.4067 /$ S0718-07642014000300019

\title{
Análisis Termodinámico de un Sistema de Absorción Tipo GAX Enfriado por Aire, para Acondicionamiento Térmico de Espacios en Climas Cálidos Extremos
}

\author{
Yair Castro-García ${ }^{(1) \star}$, Nicolás Velázquez-Limón ${ }^{(2)}$, Rafael Villa-Angulo(3) y Daniel Sauceda-Carvajal ${ }^{4}$ \\ Universidad Autónoma de Baja California (UABC), $(1,2,3)$ Instituto de Ingeniería, (4) Facultad de \\ Ingeniería, Calle de la normal S/N y Blvd. Benito Juárez, CP 21280, Col. Insurgentes Este, Mexicali B. C.- \\ México. (e-mail: yair.castro.garcia@gmail.com, nicolas.velazquez@uabc.edu.mx, rafael.villa@uabc.edu.mx, \\ daniel.sauceda@uabc.edu.mx)
}

Recibido Dic. 5, 2013; Aceptado Feb. 19, 2014; Versión final recibida Feb. 21, 2014

\begin{abstract}
Resumen
Este trabajo presenta la simulación y análisis termodinámico de un sistema de enfriamiento por absorción avanzado tipo GAX enfriado por aire. El estudio del sistema se hace para acondicionamiento térmico de espacios de sectores residenciales localizados en regiones con climas cálidos extremos. El modelo matemático del sistema se resuelve usando un algoritmo computacional de "búsqueda hacia atrás" (backtracking). Se desarrolló un simulador por medio del cual se evaluó el comportamiento operativo del sistema, bajo diferentes condiciones ambientales. Se encontró que la temperatura ambiente influye de manera importante en el comportamiento operativo del sistema, debido a que la eficiencia disminuye $20.4 \%$ al pasar de 40 a $50^{\circ} \mathrm{C}$. Se concluye que el sistema de enfriamiento GAX operado en climas cálidos extremos es una buena opción para el acondicionamiento térmico de espacios ya que tiene un alto coeficiente de operación comparado con sistemas convencionales
\end{abstract}

Palabras clave: absorción, sistema GAX, análisis termodinámico, simulación, climas calientes extremos

\section{Thermodynamic Analysis of a GAX Absorption System Air- Cooled Type for Thermal Conditioning Spaces in Extreme Hot Weather}

This paper presents the simulation and thermodynamic analysis of an absorption cooling advanced system of the type air-cooled GAX. The study of the system is focused on the thermal conditioning of residential spaces located in regions with extreme hot weather. The mathematical model of the system is solved using a computational algorithm called backtracking. A simulator was developed to evaluate the operational behavior of the system, under different environmental conditions. It was found that the ambient temperature has an important influence on the operating behavior of the system, since efficiency decreased by $20.4 \%$ when the temperature changed from 40 to $50^{\circ} \mathrm{C}$. It is concluded that the GAX cooling system operated in hot extreme weather is a good alternative option for the thermal conditioning of spaces since it maintains a high performance coefficient compared to conventional systems.

Keywords: absorption, GAX system, thermodynamic analysis, simulation, extreme hot weather 


\section{INTRODUCCIÓN}

El consumo de energía eléctrica es una causa indirecta de contaminación ambiental que contribuye al efecto invernadero y calentamiento global del planeta, sin embargo es indispensable para cubrir necesidades de acondicionamiento térmico de espacios en sectores residenciales ubicados en regiones de climas cálidos extremos con temperaturas alrededor de $50^{\circ} \mathrm{C}$ en los meses de verano, por ejemplo el Valle de Mexicali situado en la península de Baja California, México (32 $38^{\prime}$ Norte y $115^{\circ} 20^{\prime}$ Oeste). Para ayudar a contrarrestar los problemas ambientales y satisfacer las necesidades de climatización de espacios en forma simultánea, es necesario migrar hacia sistemas de enfriamiento sustentables que utilicen formas de energía limpia en lugar de la convencional. En este sentido, el potencial de los sistemas de enfriamiento por absorción se encuentra en la disminución del consumo de energía eléctrica dentro y fuera de las horas pico, utilizan calor residual, incrementan la eficiencia de los sistemas de cogeneración al generar energía eléctrica, térmica y frigorífica simultáneamente, protegen el medio ambiente porque pueden ser activados con energía solar, energía geotérmica, gas natural o biomasa (biogás) y además beneficia la economía del usuario.

En particular el sistema avanzado de enfriamiento por absorción con intercambio de calor entre el Generador y el Absobedor (GAX, por sus siglas en inglés) se caracteriza por su alto coeficiente de operación (COP) debido a su alta integración energética interna. Algunos estudios teóricos y experimentales de este sistema se enfocan en la selección de nuevos fluidos de trabajo, mejorar la transferencia de calor y masa, desarrollo de unidades más compactas y eficientes, diseño de nuevos ciclos, optimización por simulación numérica, entre otros. Phillips (1990) compara varios sistemas de absorción avanzados que funcionan con diferentes fluidos de trabajo y concluye que el más aceptable para el acondicionamiento de espacios en el sector residencial, es el ciclo GAX que trabaja con amoniaco-agua. Grossman y Wilk (1994) estudiaron a través de simulación numérica varias configuraciones de ciclos que operan con los fluidos de trabajo bromuro de litio-agua y amoniaco-agua, los cuales son activados con energía solar; para la resolución de los modelos matemáticos aplican el enfoque basado en ecuaciones. Mejoran la capacidad de convergencia en ciclos complejos.

Engler et al. (1997) comparan teóricamente el desempeño de varios ciclos de absorción (amoniaco-agua). Resuelven el modelo matemático de manera simultánea. Encontraron que el ciclo GAX enfriado por agua tiene un COP de 1.0. Grossman y Zaltash (2001) presentan el código ABSIM para simular sistemas de absorción que operan con diferentes fluidos de trabajo. El sistema de ecuaciones se resuelve en forma simultánea; Mejoran la capacidad de convergencia para ciclos complejos como el GAX, sin embargo para ciertas condiciones de operación siguen teniendo problemas de convergencia. Velázquez y Best (2002) proponen un ciclo GAX para climatización residencial en lugares con climas de temperaturas de $40^{\circ} \mathrm{C}$, con una capacidad de enfriamiento de $10.6 \mathrm{~kW}$ (3 Ton), es activado con energía solar y gas natural. Desarrollaron una metodología que contempla una secuencia de cálculo para las corrientes externas y un procedimiento iterativo para las corrientes internas. En el estudio de simulación se encontró un COP de 0.86 para enfriamiento y de 1.86 para calentamiento. Horuz y Callander (2004) evaluaron el desempeño de un sistema de refrigeración por absorción comercialmente disponible para aplicaciones de aire acondicionado; el sistema es activado por gas, utiliza amoniaco-agua como fluido de trabajo, tiene una capacidad de enfriamiento de $10 \mathrm{~kW}$. Efectuaron pruebas con el condensador-absorbedor enfriados por aire, también con el condensador enfriado por agua y el absorbedor enfriado por aire. Estudiaron el comportamiento termodinámico del sistema bajo diferentes condiciones de operación.

Kumar y Udayakumar (2007) presentan el estudio teórico sobre un ciclo (amoniaco-agua) hibrido de absorción-compresión con intercambio de calor entre el generador y el absorbedor (GAXAC) para aire acondicionado; comparan el ciclo hibrido con un ciclo GAX convencional y encuentran que el ciclo hibrido es $30 \%$ más eficiente. Gómez et al. (2008) presentan la evaluación teórica y experimental del prototipo de un sistema GAX (GAX-PCS) para aire acondicionado, con capacidad de enfriamiento de $10.6 \mathrm{~kW}$, usa amoniaco agua como fluido de trabajo y es activado indirectamente por fuego. Concluyen que el GAX-PCS tiene potencial para competir en el mercado Mexicano. Rameshkumar et al. (2009) reportan el modelo de transferencia de calor de un ciclo GAXAC; concluyen que la transferencia de calor en el absorbedor y las altas temperaturas en el generador influyen de manera importante en la eficiencia y capacidad del ciclo. Simulan el ciclo con el enfoque orientado a ecuaciones. Jawahar y Saravanan (2010) presentan el estado del arte de los ciclos GAX y explican a detalle sus diferentes configuraciones; enfatizan la ventaja de su alto desempeño comparado con los ciclos de absorción convencionales de simple efecto que trabajan con el fluido amoniaco-agua.

La revisión de los antecedentes muestra que el sistema GAX tiene potencial para acondicionamiento térmico de espacios en condiciones ambientales con temperaturas menores a $40^{\circ} \mathrm{C}$. Debido a las condiciones climatológicas extremas que se registran en diferentes partes del mundo, es necesario estudiar 
el comportamiento del ciclo en condiciones de temperatura mayores. Por lo anterior, en el presente trabajo se presenta la simulación y análisis termodinámico de un sistema de enfriamiento por absorción avanzado tipo GAX que utiliza amoniaco-agua como fluido de trabajo y es enfriado por aire, aplicado en acondicionamiento térmico de espacios localizados en regiones con climas cálidos extremos (temperaturas de 40 a $\left.50^{\circ} \mathrm{C}\right)$.

\section{DESCRIPCIÓN DEL CICLO AVANZADO GAX}

El sistema de enfriamiento por absorción tipo GAX (Figura 1) utiliza la solución amoniaco-agua como fluido de trabajo, es enfriado por aire y es activado con biogás que proviene de los desechos orgánicos de las viviendas (Maghanaki et al. 2013), en Morero y Campanella (2012) y Souza y Schaeffer (2013) se aprecia el potencial de este biocombustible. El ciclo está compuesto por un condensador (CO), un evaporador (EV), un preenfriador (PE), una columna del generador dividida en un rectificador (RE), un intercambiador calentado con solución (GHX) e intercambiador calentado por fuego directo (GBG) que utiliza biogás, una columna del absorbedor integrada por un intercambiador de calor entre el absorbedor y generador (GAX), un intercambiador de calor enfriado por la solución $(A H X)$ y la sección del absorbedor enfriada por aire $(A B)$, y por dos válvulas de expansión que dividen la zona de baja y alta presión (VER y VES). La integración energética interna se realiza en el PE, GHX, AHX y GAX.

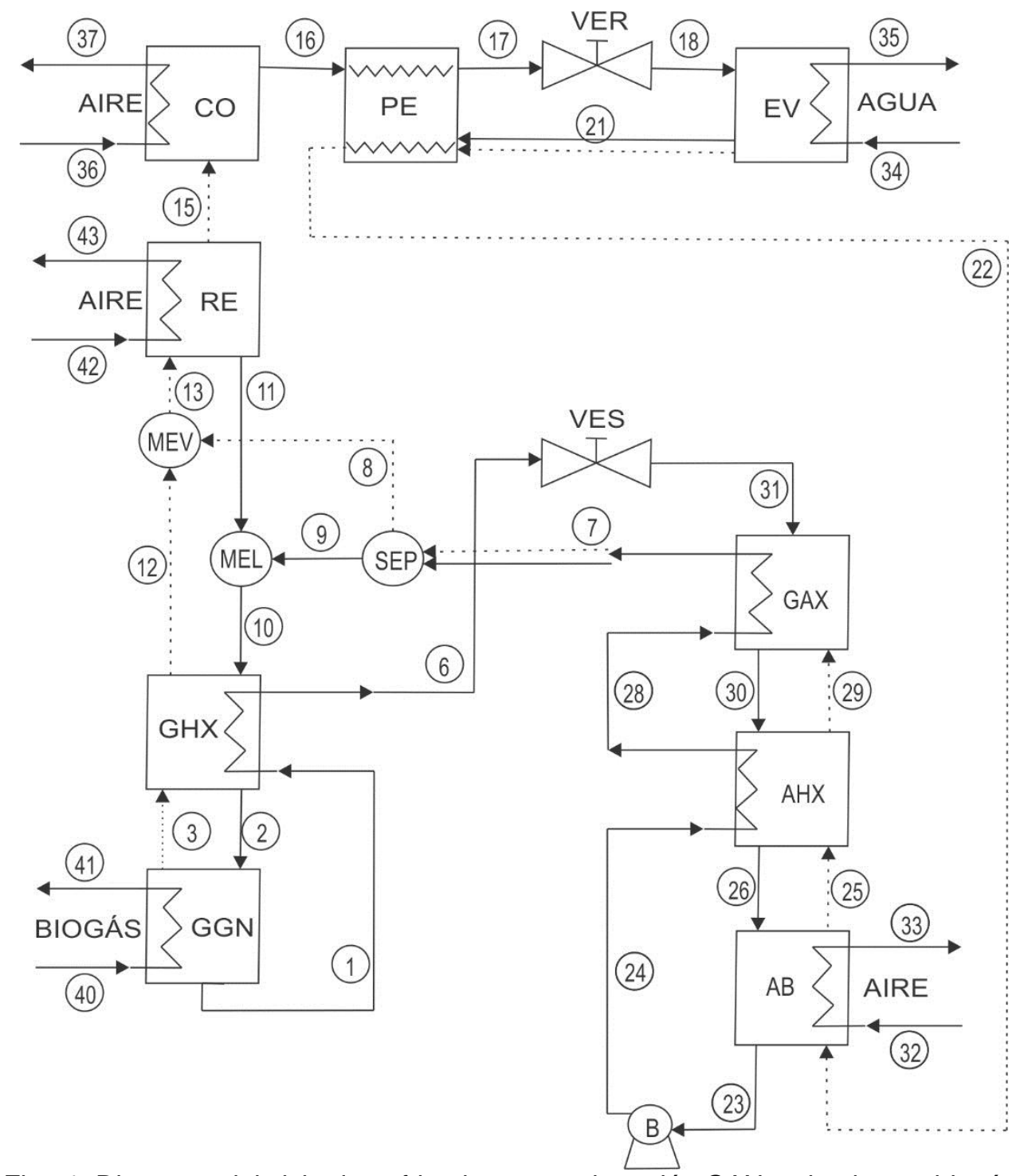

Fig. 1: Diagrama del ciclo de enfriamiento por absorción GAX activado con biogás.

En el estado 15 sale vapor de amoníaco saturado a la presión alta del sistema y se condensa en el condensador para salir como líquido saturado (16); se subenfria en el pre-enfriador (17) y se expande (dos fases) al reducir la presión en la válvula de estrangulamiento (18); el refrigerante recibe calor del agua a enfriar en el evaporador y termina de convertirse en vapor, produciendo el efecto frigorífico deseado y sale en forma de vapor saturado (21) o en algunos casos con un poco de líquido; este líquido se termina de 
evaporar en el pre-enfriador (22). El vapor frío entra luego a la columna absorbedor-GAX donde se absorbe en tres diferentes etapas por medio de una solución acuosa caliente; en el absorbedor se retira el calor de la reacción exotérmica (generada por el fluido de amoniaco-agua) por medio de aire de enfriamiento y por usar la misma solución fría que deja el absorbedor; la solución acuosa con alto porcentaje de amoníaco en agua (solución fuerte) sale de la columna absorbedor-GAX (23) y entra en la bomba (24); a presión alta del sistema se introduce a la sección media de la columna (AHX), donde recibe calor del absorbedor, saliendo en el estado 28.

Posteriormente entra en la parte más caliente del absorbedor (GAX), en donde al recibir calor de absorción de alta calidad, alcanza el punto de saturación e inicia el proceso de cambio de fase (liberación de amoníaco), sale en el estado 7, como fluido vapor-líquido entra en la cámara de separación de la columna generador-rectificador, en donde la parte líquida se incorpora al vapor condensado proveniente del rectificador (11) y entran al generador (10), en donde se agrega calor para terminar de extraer el amoníaco de la solución. El líquido caliente (solución débil), sale por el fondo de la columna generador-rectificador (1); posteriormente la solución caliente se introduce de nuevo a la columna para que caliente la sección GHX del generador, saliendo en el estado 6. Luego reduce su presión al pasar por una válvula, para salir en el estado 31. La solución acuosa todavía caliente entra a la columna absorbedor-GAX por la parte superior, en donde se pone en contacto en contra corriente con el vapor de amoníaco a absorber; regresando al generador, la liberación del amoníaco en forma de vapor se realiza en tres etapas y sale del generador (12), pasando a la cámara de separación en donde se une con la fase vapor proveniente de la columna absorbedor-GAX (13). La corriente de vapor ascendente, sale de la cámara de separación y entra al rectificador en donde se le retira calor, saliendo después amoniaco de alta pureza en el estado 15.

De esta manera se completa la operación del ciclo. Se asume que el sistema: opera en estado estable, la solución amoniaco-agua se encuentran en equilibrio, no existe caída de presión debido a la fricción, excepto en la válvula de expansión, el proceso de expansión de los fluidos al pasar por las válvulas del sistema es isoentálpico, el sistema se considera adiabático, se desprecian los cambios en la energía potencial y energía cinética, los equipos: generador, condensador, evaporador y absorbedor trabajan en condiciones de saturación.

\section{MÉTODO DE SOLUCIÓN}

El ciclo GAX es representativo del resto de los ciclos avanzados de enfriamiento por absorción debido a su nivel de integración energética interna, sin embargo esta característica provoca que el modelo matemático que lo representa sea no lineal, con alta complejidad de resolución (converger a una solución). En esta sección se presenta un método de solución que incorpora el algoritmo computacional backtracking. De manera general, el algoritmo backtracking asigna un valor a la variable actual de su dominio; después se verifica la solución parcial, si alguna de las restricciones de las variables actuales o pasadas se viola, se abandona la asignación y se selecciona otro valor del dominio para asignarlo nuevamente a la variable; si se han probado todos los valores del dominio de la variable actual, el algoritmo regresa a la variable anterior y le asigna un nuevo valor; el algoritmo termina cuando se ha asignado un valor para cada variable (solución completa) o se han considerado todas las posibilidades (Tsang, 1993).

La idea esencial en la que se basa el Método de Solución (MS) del sistema, es aplicar un Estrategia de Solución (ES) al modelo matemático de una unidad del ciclo para obtener su solución numérica, y con base en esa solución, resolver el modelo matemático de una unidad siguiente; para ello se define una Secuencia $\left(S_{q}\right)$ que involucra a todas las unidades del ciclo; el algoritmo realiza la búsqueda sistemática de soluciones unidad por unidad través de la secuencia hasta encontrar la convergencia a una solución del ciclo completo. El modelo matemático que describe el comportamiento completo de cada unidad, comprende las ecuaciones termodinámicas requeridas, tales como de conservación de masa, conservación de energía, transferencia de calor y equilibrio de líquido-vapor. La Secuencia $S_{q}$ se define con base a los datos disponibles en el modelo matemático de cada unidad, la meta es resolver primero aquellas unidades que cuentan con mayor cantidad de datos, por lo tanto la Secuencia no refleja necesariamente la adyacencia de las unidades dentro del ciclo.

La Estrategia de Solución ES de una unidad, inicia por ordenar el modelo matemático en orden descendente con base en la mayor cantidad de variables que se conocen o que se pueden calcular de forma directa (Figura 2). Después se calculan las variables únicamente por sustituir valores o calcularlas con algún método matemático factible, la meta es asignar un valor a la mayor cantidad de variables. Si ya no restan variables para calcular (todas las variables tienen un valor asignado), se procede a verificar en el modelo matemático que se cumplan las restricciones y la igualdad de las ecuaciones con una tolerancia de error $\left(\varepsilon_{r}\right)$, es decir se obtiene una solución factible y la estrategia termina; en caso de que falte calcular algunas variables, se identifica un conjunto de variables iterativas $\left(V_{i}\right)$, un dominio $\left(D_{i}\right)$ factible para cada una 
de ellas y un incremento $\left(\varepsilon_{i}\right)$ para recorrer cada dominio; las variables iterativas deben permitir que se calculen otras variables a partir de ellas, el dominio se define con base a las cotas superiores e inferiores que no pueden rebasar las variables iterativas, y el intervalo se debe seleccionar acorde al impacto que cause en la igualdad de las ecuaciones del modelo matemático. Utilizando $\varepsilon_{i}$ se asigna un valor a cada variable iterativa (combinación de valores); pueden existir diferentes conjuntos de variables iterativas, sin embargo es mejor elegir el que contiene menor cantidad de ellas, porque de esta manera se generan menos combinaciones. Como consecuencia, es posible calcular las variables restantes y se procede a verificar la igualdad de las ecuaciones en el modelo matemático, si no se satisface, entonces se asigna una combinación nueva de valores a las variables iterativas (previamente se verifica que el dominio sea válido) hasta que se obtenga una solución de la unidad. La Estrategia de Solución finaliza cuando se obtiene una solución de la unidad o cuando se ha agotado el dominio valido de las variables iterativas.

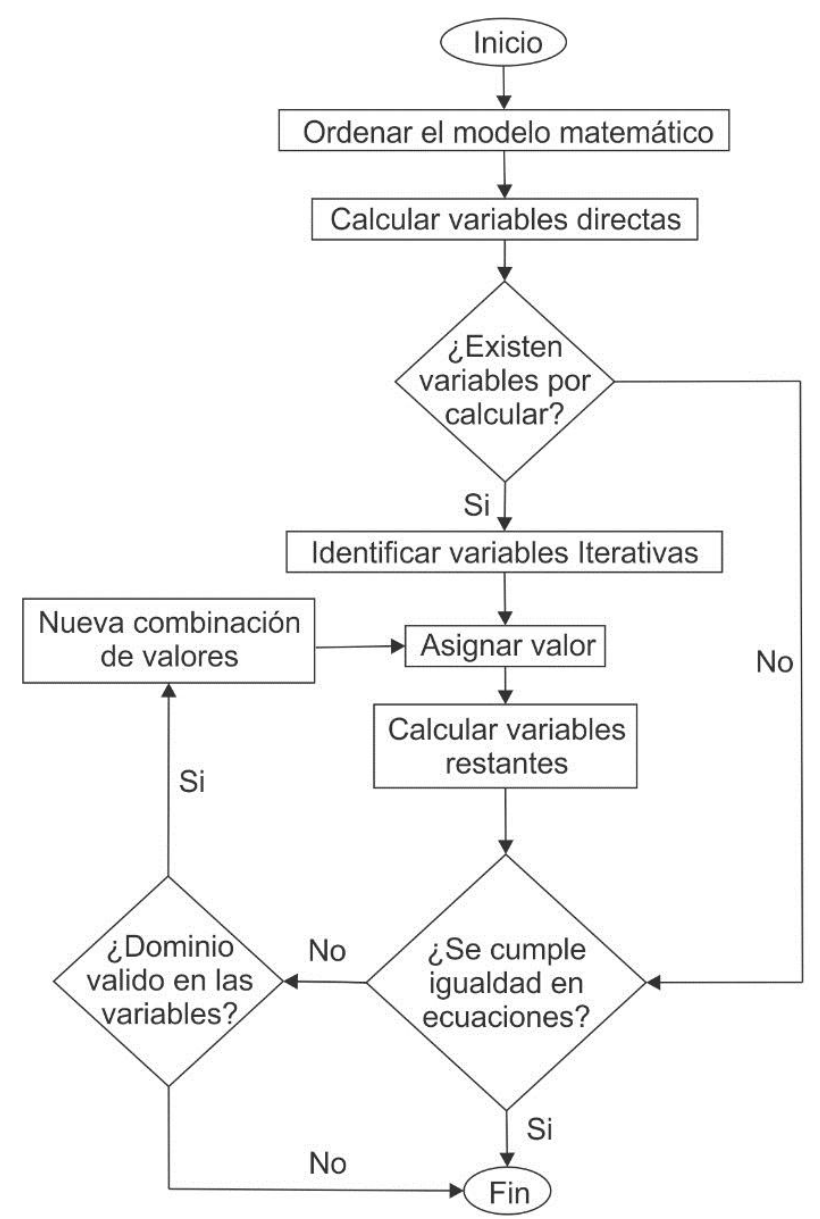

Fig. 2: Estrategia de Solución (ES) para resolver el modelo matemático de una unidad del sistema.

La Figura 3 ilustra el Método de Solución MS para resolver el modelo matemático que representa al ciclo GAX en forma completa. Inicia recibiendo la Secuencia $\left(S_{q}\right)$ de unidades y la longitud $n$ de la misma, enseguida el algoritmo backtracking inicializa el contador ( $I$ ) con el valor de 1. Después selecciona la unidad $M_{i}$ de la Secuencia $\left(S_{q}\right)$ y aplica la Estrategia de Solución ES asumiendo que el ordenamiento del modelo matemático, el cálculo de las variables en forma directa y la identificación de las variables iterativas se hace una sola vez. En lugar de finalizar la Estrategia de Solución, el algoritmo guarda la solución de la unidad en un vector $(C)$ de longitud máxima $n$, en donde cada posición en el vector corresponde a la solución de cada unidad $\left(M_{i}\right)$. Posteriormente verifica si la unidad actual $\left(M_{i}\right)$ es la última $(i=n)$ en la Secuencia, si es así imprime la solución $(C)$ completa del ciclo y el Método de Solución termina. En caso contrario el algoritmo verifica si el dominio $(D)$ de las variables iterativas relacionadas a la unidad en curso $\left(M_{i}\right)$ se han recorrido por completo, en caso de que no, incrementa el contador (i) para seguir aplicando la estrategia ES a la siguiente unidad en la Secuencia $S_{q}$, es importante notar que la solución de la unidad en curso aplica la estrategia ES tomando en cuenta la solución de las corrientes de la unidad anterior en el ciclo, esto asegura soluciones mutuamente compatibles entre unidades y una solución factible para el ciclo completo. En el caso de haber recorrido el dominio completo de todas las variables iterativas relacionadas a la unidad en curso $\left(D\left(M_{i}\right)>\right.$ Lsup $)$, se reinicia el dominio de sus variables $\left(R D\left(M_{i}\right)\right)$ y se decrementa el contador $(I)$ para seguir aplicando la estrategia ES a la unidad anterior en la secuencia $\left(S_{q}\right)$. 


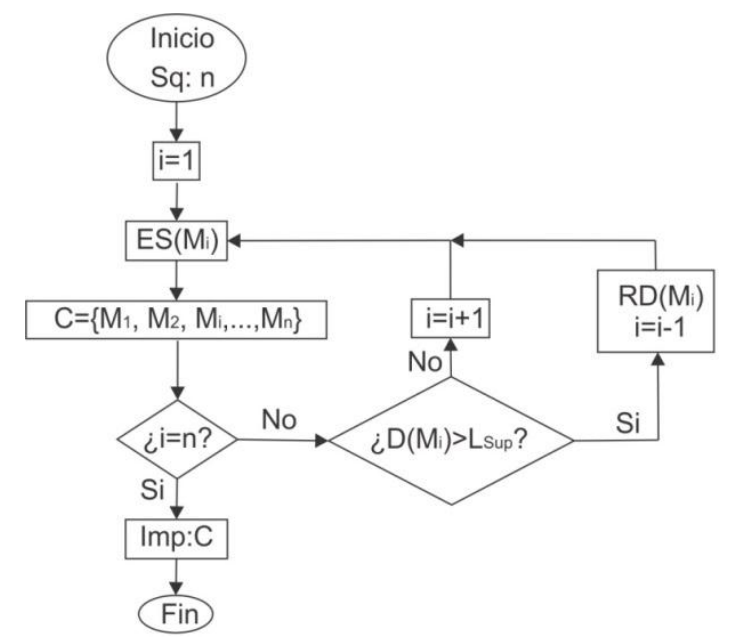

Fig. 3: Método de Solución (MS) del sistema GAX.

Una vez que se puede resolver numéricamente el ciclo GAX a través del Método de Solución, se desarrolla un simulador de tipo específico. La Figura 4 ilustra las etapas en que el simulador SIMSABA (Simulador de sistemas de absorción avanzados) simula al ciclo GAX. El usuario interactúa con el simulador por medio de una interfaz gráfica implementada en el sistema operativo MS-Windows; proporciona parámetros y condiciones de operación bajo las que se simulan al ciclo; de esta manera, el orden de las unidades en la Secuencia $\left(S_{q}\right)$, las variables iterativas y el Método de Solución son transparentes para el usuario.

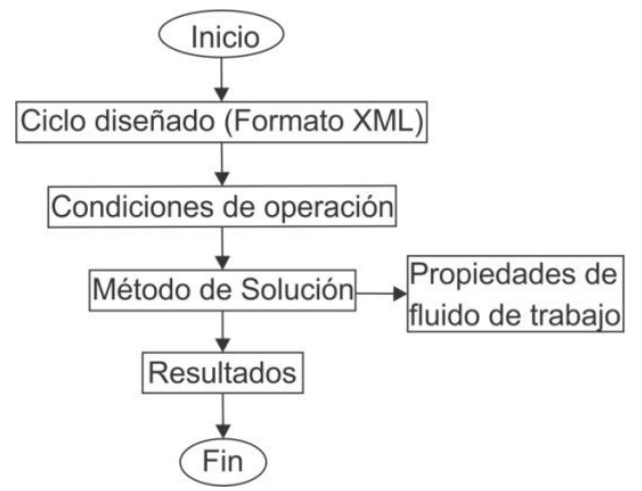

Fig. 4: Diagrama del simulador.

El usuario inicia una simulación cargando el ciclo diseñado previamente que se encuentra almacenado en un archivo con formato XML, el diagrama del ciclo se despliega en la interfaz gráfica en donde se muestran las unidades y corrientes del ciclo. Después el usuario proporciona las condiciones de operación bajo las cuales desea simular al ciclo. La interfaz permite seleccionar una unidad del ciclo y se despliegan automáticamente sus características, como el calor transferido, propiedades de cada corriente y su descripción en las unidades correspondientes. Dentro del simulador se encuentra codificado el Método de Solución anteriormente descrito e incorpora las propiedades del agua como lo indica ASHRAE (1997) y las correlaciones de Patek y Klomfar (1995) para la solución amoniaco-amoniaco. Finalmente, los estudios de simulación permiten conocer la temperatura, entalpia, flujo másico, concentración, presión y fracción de vapor, en cada corriente del ciclo para diferentes parámetros de diseño; también se calcula el calor transferido en cada unidad y el COP del sistema. Los resultados de una simulación se pueden ver en la interfaz gráfica y se guardan de manera automática en formato de Excel para facilitar su análisis a través de formatos enriquecidos.

\section{RESULTADOS DE LA SIMULACIÓN}

La simulación numérica del ciclo de enfriamiento por absorción avanzado GAX para acondicionamiento de espacios en el sector residencial localizados en regiones con climas cálidos extremos se efectúa bajo las siguientes condiciones de diseño: el sistema trabaja con el fluido amoniaco-agua, es activado con biogás, tiene una capacidad de enfriamiento de $10.5 \mathrm{~kW}$ (3 Ton), 97\% de líquido a la entrada del evaporador, la concentración del refrigerante en la solución débil a la salida del generador es de $4 \%$ y la concentración del 
refrigerante en el vapor que entra al condensador es de 99.3\%. En la simulación (estudio paramétrico) se cambia una condición de operación en forma gradual mientras el resto de los parámetros se mantienen fijos. Se investiga la temperatura ambiente en el rango de 40 a $50^{\circ} \mathrm{C}$ con incrementos de $5^{\circ} \mathrm{C}$, sobre la condición estándar de temperatura en el agua fría del evaporador (entra a $12^{\circ} \mathrm{C}$ y sale a $7^{\circ} \mathrm{C}$ ), como parte de la exploración también se evaluó con una entrada de $15^{\circ} \mathrm{C}$ y salida de $10^{\circ} \mathrm{C}$, manteniendo fija siempre la capacidad de enfriamiento del sistema. El coeficiente de operación para enfriamiento (COPEnf) se define como la razón del calor que retira el evaporador entre el calor suministrado en el generador, y el coeficiente de operación para calentamiento (COPCal) se define como la suma del calor rechazado en el rectificador, condensador y absorbedor entre el calor suministrado en el generador. La Secuencia de resolución de las unidades que resulta de aplicar el Método de Solución al ciclo, se conforma de la siguiente manera: EV, CO, PE, VER, GBG, RE, MEL, MEV, SEP, GHX, VES, GAX, BS, AHX y AB; las variables iterativas son: T2, T12 y T25 con incrementos $\varepsilon_{i}=0.1$ y $\varepsilon_{r}=0.001$.

La temperatura ambiente de 45 y $50^{\circ} \mathrm{C}$ combinada con la temperatura a la salida del evaporador $\left(7\right.$ y $\left.10^{\circ} \mathrm{C}\right)$ generan las temperaturas Lift (diferencia de temperatura del condensador y el evaporador) de 48, 51, 53 y $56^{\circ} \mathrm{C}$, provocando que el sistema GAX trabaje a presión por encima de 20 bar, y dado que las ecuaciones para el cálculo de propiedades de Patek y Klomfar (1995), integradas en el simulador SIMSABA, son recomendadas para trabajar en un rango de operación inferior ( $0.002 \mathrm{Mpa}(0.02$ bar) hasta $2 \mathrm{MPa}(20 \mathrm{bar})$ ), es necesario hacer un estudio comparativo con las propiedades obtenidas en el paquete REFPROP (Lemmon et al. 2007) fuera del rango mencionado. Analizando el comportamiento de la relación $T(p, x)$ y comparando la tendencia con temperaturas ambiente mayores a $40^{\circ} \mathrm{C}$, la Figura 5 muestra que no hay diferencia en el comportamiento de los datos generados por ambas correlaciones para una concentración constante de amoniaco (99.3\%) en el condensador. Sin embargo es necesario aplicar una prueba estadística a la muestra (presión) generada con la relación $\mathrm{T}(\mathrm{p}, \mathrm{x})$ para definir si los valores son diferentes en forma significativa al compararse con la muestra generada en REFPROP. Las dos muestras son de tamaño $n=40$, independientes porque provienen de distintos software (poblaciones), no se afectan entre si y no se asume que sigan una distribución normal, por lo tanto se aplica la prueba de Wilcoxon (Siegel y Castellana 1995) con un nivel de significancia de 0.05 para decidir si las distribuciones son idénticas.

Se contrasta la hipótesis nula $\left(H_{0}\right)$ con la hipótesis alternativa $\left(H_{1}\right)$ :

$H_{0}$ : No existe diferencia en la mediana de ambas muestras de la presión con la hipótesis alternativa.

$H_{1}$ : La diferencia en las medianas de ambas muestras de presión es mayor a cero.

La prueba arroja un valor estadístico de 0.1379 que es mayor al nivel de significancia, por lo tanto no se rechaza $H_{0}$. La aceptación de la hipótesis nula permite concluir se puede confiar en la relación $T(p, x)$ de Patek y Klomfar, (1995) para determinar las propiedades termodinámicas de presión y temperatura en el sistema GAX simulado.

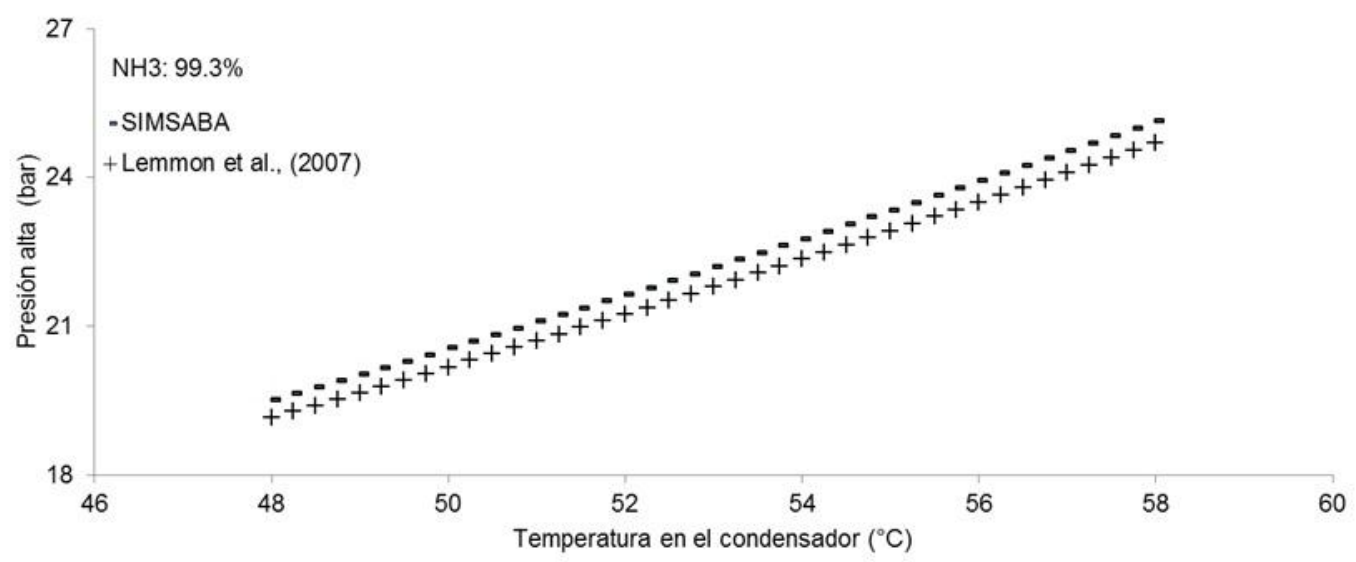

Fig. 5: Comportamiento de la relación $\mathrm{T}(\mathrm{p}, \mathrm{x})$ con temperaturas ambiente de 40 a $50^{\circ} \mathrm{C}$.

En la Tabla 1 se muestra los resultados de la simulación para diferentes temperaturas Lift del ciclo; simultáneamente con la Figura 6 se observa que el COP de enfriamiento y COP de calentamiento disminuyen conforme incrementa la temperatura ambiente (incremento de la temperatura Lift del ciclo); comparando los dos COP que representan a las temperaturas Lift menor y mayor, se observa una disminución de $30 \%$ en modo de operación de enfriamiento y $12 \%$ en modo de calentamiento, lo cual se debe a que el incremento de la temperatura del aire de enfriamiento (ambiente) y respetando un gradiente de temperatura con respecto al refrigerante en el condensador, se provoca un incremento en la presión del 
sistema en la zona de alta, esto dificulta la liberación del refrigerante en el generador, requiriéndose mayor suministro de energía, e impactando directamente en la eficiencia del sistema.

Tabla 1: Resultados de la simulación para el ciclo avanzado GAX bajo diferentes condiciones de operación.

\begin{tabular}{|c|c|c|c|c|c|c|c|c|c|c|c|c|c|}
\hline T. Lift $\left({ }^{\circ} \mathrm{C}\right)$ & 43 & 46 & 48 & 51 & 53 & 56 & T. Lift $\left({ }^{\circ} \mathrm{C}\right)$ & 43 & 46 & 48 & 51 & 53 & 56 \\
\hline \multicolumn{8}{|c|}{ Condensador } & \multicolumn{6}{|c|}{ Evaporador } \\
\hline $\mathrm{T} 15\left({ }^{\circ} \mathrm{C}\right)$ & 69 & 69 & 73 & 73 & 76 & 76 & $\mathrm{~T} 18\left({ }^{\circ} \mathrm{C}\right)$ & 5 & 2 & 5 & 2 & 5 & 2 \\
\hline $\mathrm{T} 16\left({ }^{\circ} \mathrm{C}\right)$ & 48 & 48 & 53 & 53 & 58 & 58 & $\mathrm{~T} 21\left({ }^{\circ} \mathrm{C}\right)$ & 9 & 6 & 9 & 6 & 9 & 6 \\
\hline T36 ( $\left.{ }^{\circ} \mathrm{C}\right)$ & 40 & 40 & 45 & 45 & 50 & 50 & T34 $\left({ }^{\circ} \mathrm{C}\right)$ & 15 & 12 & 15 & 12 & 15 & 12 \\
\hline T37 $\left({ }^{\circ} \mathrm{C}\right)$ & 47 & 47 & 52 & 52 & 57 & 57 & T35 $\left({ }^{\circ} \mathrm{C}\right)$ & 10 & 7 & 10 & 7 & 10 & 7 \\
\hline QCO (kW) & 10.6 & 10.5 & 10.4 & 10.3 & 10.2 & 10.1 & QEV $(\mathrm{kW})$ & 10.6 & 10.6 & 10.6 & 10.6 & 10.6 & 10.6 \\
\hline \multirow[t]{2}{*}{ m16 (Kg/Min) } & 0.55 & 0.55 & 0.55 & 0.55 & 0.55 & 0.55 & & \multicolumn{6}{|c|}{ Resto del ciclo GAX } \\
\hline & \multicolumn{6}{|c|}{ Absorbedor } & T6, T31 $\left({ }^{\circ} \mathrm{C}\right)$ & 121 & 123 & 130 & 130 & 137 & 135 \\
\hline T22 $\left({ }^{\circ} \mathrm{C}\right)$ & 42 & 42 & 47 & 47 & 52 & 52 & T7-T13 $\left({ }^{\circ} \mathrm{C}\right)$ & 115 & 117 & 124 & 124 & 131 & 129 \\
\hline T23 $\left({ }^{\circ} \mathrm{C}\right)$ & 48 & 48 & 53 & 53 & 58 & 58 & $\mathrm{~T} 17\left({ }^{\circ} \mathrm{C}\right)$ & 18 & 17 & 22 & 22 & 27 & 26 \\
\hline T25, T26 ( $\left.{ }^{\circ} \mathrm{C}\right)$ & 78 & 80 & 85 & 88 & 94 & 99 & T24 $\left({ }^{\circ} \mathrm{C}\right)$ & 48 & 48 & 53 & 53 & 58 & 58 \\
\hline T32 $\left({ }^{\circ} \mathrm{C}\right)$ & 40 & 40 & 45 & 45 & 50 & 50 & $\mathrm{~T} 28\left({ }^{\circ} \mathrm{C}\right)$ & 99 & 103 & 111 & 115 & 122 & 126 \\
\hline T33 $\left({ }^{\circ} \mathrm{C}\right)$ & 46 & 46 & 51 & 51 & 56 & 56 & T29, T30 ( $\left.{ }^{\circ} \mathrm{C}\right)$ & 105 & 109 & 117 & 121 & 128 & 132 \\
\hline m23 (Kg/Min) & 1.28 & 1.34 & 1.38 & 1.44 & 1.49 & 1.56 & QPE $(k W)$ & 1.3 & 1.4 & 1.4 & 1.4 & 1.4 & 1.4 \\
\hline \multirow[t]{2}{*}{ QAB $(\mathrm{kW})$} & 8.3 & 8.8 & 8.8 & 9.6 & 9.7 & 11.3 & QRE (kW) & 2.9 & 3.1 & 3.5 & 3.5 & 3.9 & 3.7 \\
\hline & \multicolumn{6}{|c|}{ GBG } & QGAX (kW) & 5.5 & 4.9 & 4.9 & 3.8 & 3.5 & 1.3 \\
\hline $\mathrm{T} 1\left({ }^{\circ} \mathrm{C}\right)$ & 199 & 199 & 205 & 205 & 212 & 212 & QAHX (kW) & 5.0 & 5.6 & 6.1 & 6.8 & 7.4 & 8.2 \\
\hline T2, T3 $\left({ }^{\circ} \mathrm{C}\right)$ & 136 & 139 & 147 & 148 & 156 & 154 & QGHX (kW) & 4.3 & 4.5 & 4.8 & 5.1 & 5.5 & 6.1 \\
\hline $\mathrm{T} 40\left({ }^{\circ} \mathrm{C}\right)$ & 239 & 239 & 245 & 245 & 252 & 252 & P. Alta (bar) & 19.5 & 19.5 & 22.2 & 22.2 & 25.2 & 25.2 \\
\hline $\mathrm{T} 41\left({ }^{\circ} \mathrm{C}\right)$ & 193 & 192 & 198 & 194 & 199 & 194 & P. Baja (bar) & 5.2 & 4.7 & 5.2 & 4.7 & 5.2 & 4.7 \\
\hline m1 (Kg/Min) & 0.73 & 0.79 & 0.83 & 0.89 & 0.94 & 1.02 & COPEnf & 0.91 & 0.88 & 0.86 & 0.81 & 0.78 & 0.70 \\
\hline QGBG (kW) & 11.6 & 12.0 & 12.2 & 13.1 & 13.5 & 15.0 & COPCal & 1.88 & 1.87 & 1.86 & 1.78 & 1.76 & 1.67 \\
\hline
\end{tabular}

Dados los resultados y bajo las condiciones estándar en el evaporador (temperaturas Lift de 46 y $56{ }^{\circ} \mathrm{C}$ ), se determina que la temperatura ambiente influye de manera importante en el comportamiento operativo del sistema en modo de enfriamiento, debido a que la eficiencia disminuye en $20.4 \%$ al pasar de 40 a $50{ }^{\circ} \mathrm{C}$; para las mismas condiciones en el modo de calentamiento, la eficiencia disminuye en $10.6 \%$. En el caso de las temperaturas Lift de 43 y $53^{\circ} \mathrm{C}$, la eficiencia disminuye en $14.3 \%$ en modo de enfriamiento y $6.4 \%$ en modo de calentamiento. El sistema de enfriamiento GAX operado a $50^{\circ} \mathrm{C}$ y en condiciones estándar (climas cálidos extremos), es una buena opción a pesar de que muestra disminución en su eficiencia comparada con el caso de temperatura Lift de $46^{\circ} \mathrm{C}$, ya que para la unidad de $10.5 \mathrm{~kW}$ (3 Ton) de enfriamiento se logra una integración energética de $17 \mathrm{kw}$, lo cual representa un 13. 3\% más de la energía que se suministra en el generador, además de que se tiene un COP (0.7) mayor que en cualquier otro sistema convencional.

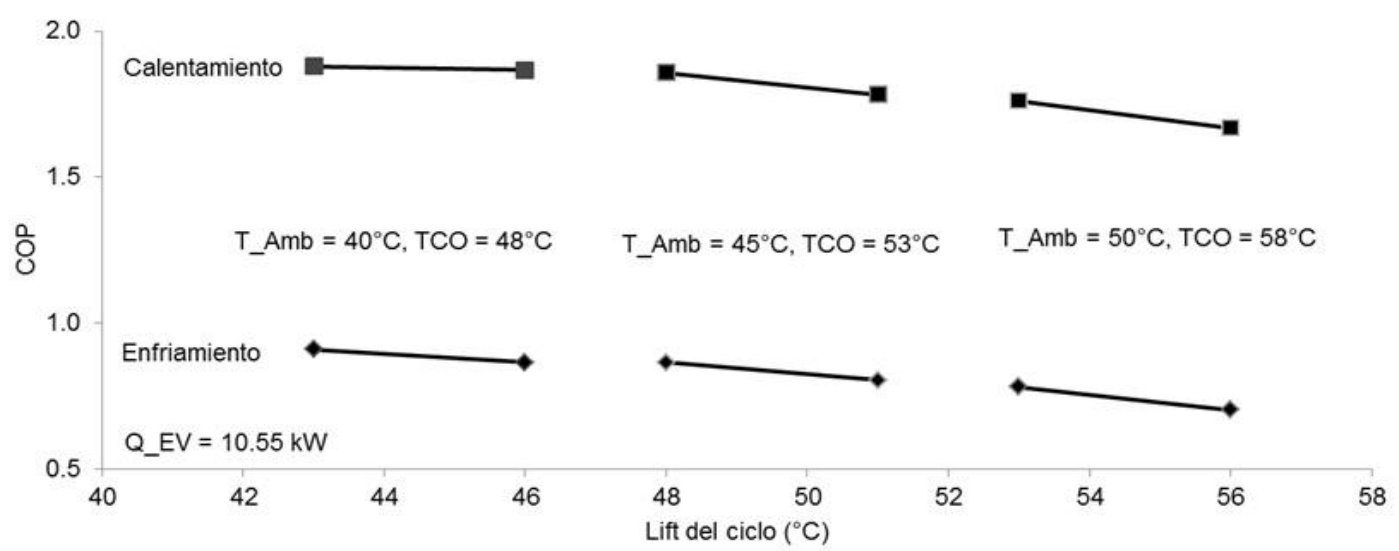

Fig. 6: Eficiencia del ciclo GAX en dos aplicaciones. 
En la Figura 7 se observa la variación del calor transferido en los componentes principales del ciclo GAX, cada segmento de línea muestra el comportamiento del calor transferido bajo diferentes condiciones de operación. Al pasar de la temperatura Lift de 46 a la de $56^{\circ} \mathrm{C}$, el calor del generador y del absorbedor se incrementa en $25 \%$ y $28.4 \%$, respectivamente, lo cual se debe al suministro de energía al sistema explicado anteriormente; bajo las mismas condiciones, el calor retirado en el sistema por las unidades $C O$, RE y $A B$ es de $22.4 \mathrm{~kW}$ y $25 \mathrm{~kW}$, el cual se puede aprovechar para procesos (por ejemplo, sacado de frutos) que se pueden establecer cerca de los espacios residenciales.

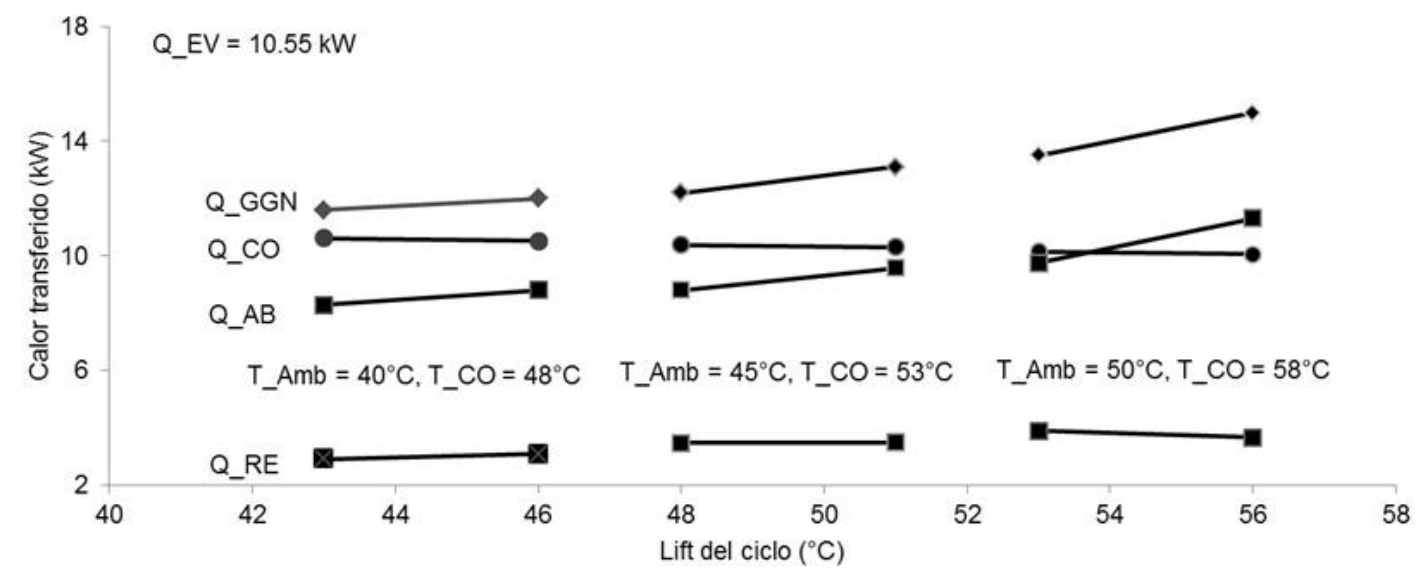

Fig. 7: Intercambio de calor en las unidades principales del ciclo GBG-GAX.

\section{CONCLUSIONES}

Con base en los resultados de la simulación y el análisis termodinámico del sistema GAX, se concluye que el sistema de enfriamiento GAX que utiliza amoniaco-agua como fluido de trabajo y enfriado por aire, es una buena opción para acondicionamiento térmico de espacios localizados en regiones con climas cálidos extremos a pesar de que muestra disminución en su eficiencia, ya que comparado con sistemas convencionales sigue teniendo alta eficiencia (COP de 0.7). El método de solución desarrollado para resolver el modelo matemático que representa al ciclo GAX muestra ser eficaz porque encuentra las soluciones (convergencia) bajo diferentes condiciones de operación. El simulador desarrollado permite estudiar el comportamiento operativo del sistema GAX y puede ser utilizado para encontrar las mejores condiciones de operación. Conforme se incrementa la temperatura ambiente de 40 a $50^{\circ} \mathrm{C}$, la eficiencia del sistema disminuye en $20.4 \%$, por lo tanto se le debe suministrar mayor energía para que logre la capacidad de enfriamiento de $10.5 \mathrm{~kW}$.

\section{REFERENCIAS}

ASHRAE Handbook, Refrigeration Systems and Applications, 2a edición, ASHRAE, Atlanta (1997).

Engler, M., G. Grossman y H. M. Helmann, Comparative Simulation and Investigation of Ammonia-Water: Absorption Cycles for Heat Pump Applications, International Journal of Refrigeration, 20(7), 504-516 (1997).

Gomez, V.H., A. Vidal, R. Best, O. Garcia-Valladares y N. Velázquez, Theoretical and Experimental Evaluation of an Indirect-Fired GAX Cycle Cooling System, Applied Thermal Engineering, 28(8-9), 985-987 (2007).

Grossman, G. y M. Wilk, Advanced Modular Simulation of Absorption Systems, International Journal of Refrigeration, 17(4), 231-244 (1994).

Grossman, G. y A. Zaltash, ABSIM - Modular Simulation of Advanced Absorption Systems, International Journal of Refrigeration, 24(6), 531-543 (2001).

Horus, I. y T.M.S. Callander, Experimental Investigation of a Vapor Absorption Refrigeration System, Int. Journal of Refrigeration, 27(1), 10-16 (2004).

Jawahar, C.P. y R. Saravanan, Generator Absorber Heat Exchange Based Absorption cycle-A Review, Renewable and Sustainable Energy Reviews, 14(8), 2372-2382 (2010). 
Lemmon, E.W., M.L. Huber y M.O. McLinden, REFPROP-thermodynamic and Transport Properties of Refrigerants and Refrigerant Mixtures, Version 8.0 (2007).

Lostec, L. B., N. Galanis y J. Millette, Experimental Study of an Ammonia-Water Absorption Chiller, International Journal of Refrigeration, 35 (0), 2275-2286 (2012).

Maghanaki Mohammadi, M., B. Ghobadian, G. Najafi y R. Janzadeh Galogah, Potential of Biogas Production in Iran, Renewable and Sustainable Energy Reviews, 28, 702-714 (2013).

Morero, B. y E. A. Campanella, Simulación del Proceso de Absorción Química con Soluciones de Aminas para la Purificación Biogás, Información Tecnológica, 24(1), 25-32 (2013).

Patek J. y J. Klomfar, Simple Functions for Fast Calculations of Selected Thermodynamic Properties of the Ammonia-Water System, International Journal of Refrigeration, 18(4), 228-234 (1995).

Phillips, B. A., Development of a High-Efficiency, Gas-Fired, Absorption Heat Pump for Residential and Small-Commercial Applications Phase 1 Final Report: Analysis of Advanced Cycles and Selection of the Preferred Cycle, Oak Ridge National Laboratory (1990).

Ramesh Kumar, A. y M. Udayakumar, Simulation Studies on GAX Absorption Compression Cooler, Energy Conversion and Management, 48(9), 2604-2610 (2007).

Ramesh kumar, A., M. Udayakumar y R. Saravanan, Heat Transfer Studies on a GAXAC (generatorAbsorber-Exchange Absorption Compression) Cooler, Applied Energy, 86(10), 2056-2064 (2009).

Siegel, S. y N. Jhon Castellana, Estadística no paramétrica: aplicada a las ciencias de la conducta, $4^{a}$ edición, 437, Trillas, D. F. México (1995).

Souza, J. y L. Schaeffer, Sistema de Compresión de Biogás y Biometano, Información Tecnológica, 24(6), 38 (2013).

Tsang, E. Foundations of Constraint Satisfaction, $1^{\text {a }}$ edición, Academic Press, 405, San Diego, CA., USA (1993).

Velázquez, N. y R. Best, Methodology for the Energy Analysis of an Air Cooled GAX Absorption Heat Pump Operated by Natural Gas and Solar Energy, Applied Thermal Engineering, 22(10), 1089-1103 (2002). 